\title{
Paraphrases, périphrases, antonomases et désignation de l'altérité
}

\author{
Véronique Magri ${ }^{1}$ \\ ${ }^{1}$ Université Côte d'Azur, CNRS, BCL, France \\ magri@unice.fr
}

\section{Résumé.}

La paraphrase a des affinités avec la périphrase en ce qu'elles servent toutes deux le processus de nomination du référent par une opération de substitution extensive, saisie à un point différent de sa réalisation. La paraphrase propose la substitution, la périphrase l'impose. $\mathrm{La}$ reformulation du nom commun exogène joue sur les deux processus. Celle du nom propre de lieux peut être assimilée à la figure de l'antonomase. Les trois processus, périphrase, paraphrase et antonomase seront envisagés dans un continuum dynamique comme spécifiques du récit de voyage. La paraphrase propose une reformulation alternative; la périphrase remplace le terme exogène par un énoncé qui assure la désignation directe. L'antonomase est plus complexe puisqu'elle désigne l'objet du monde exogène par l'emploi d'un nom propre lui-même reformulé.

\begin{abstract}
.
The paraphrase has affinities with the circumlocution because they serve both the process of denomination of the referent by an extensive substitution operation, in a point different from its realization. The paraphrase proposes the substitution, the circumlocution imposes it. The reformulation of the exogenous common noun plays on two processes. That of the toponyms can be likened to the figure of the antonomasia. Three processes, circumlocution, paraphrase and antonomasia will be
\end{abstract}


envisaged in a dynamic continuum. The paraphrase proposes an alternative reformulation; the circumlocution replaces the exogenous term by a statement which assures the direct name. The antonomasia is more complex because it indicates the object of the exogenous mode by the use of a proper noun reformulated itself.

\section{Introduction}

Depuis l'article fondateur de G. Kleiber (1984), la sémantique lexicale oppose deux processus, la dénomination et la désignation. La dénomination qui implique un présupposé référentialiste et impose une conception fixiste du lexique définit une «relation référentielle stable et récurrente», entre unités lexicales et objets du monde et s'oppose à la désignation qui propose un mode d'accès au référent non conventionnel, dépendant de l'actualisation discursive. Dans le récit de voyage, que je choisis comme observatoire expérimental pour cette étude, la relation au référent illustre ce cas particulier d'un référent pré-construit auquel est confronté le voyageur. Ce sous-genre $\mathrm{du}$ texte narratif se particularise encore par cet enjeu pragmatique fondateur qui constitue le contrat de l'énonciateur : dire l'autre, représenter le différent pour le faire percevoir ou comprendre au lecteur, resté en terre connue et avec qui il partage a priori un même réservoir lexical.

Dès lors, la description du monde découvert et l'ajustement du discours à l'altérité se déclinent en deux situations: que le référent soit inédit ou similaire à celui que connaît le voyageur-énonciateur, celui-ci peut connaître sa dénomination en langue exogène ou l'ignorer. Dans le premier cas, il devra se livrer à une traduction, dans l'autre il devra emprunter les voies linguistiques du contournement, pour donner accès au référent. Dans le contexte particulier du récit de voyage, que la dénomination soit codée en langue étrangère ou inconnue, la représentation des objets du monde s'appuie sur une perception forcément subjective et traduit une vision du monde particulière. Je choisis le processus de la reformulation pour réunir ces deux cas de figure: postulant un invariant de sens qui s'accompagne d'une variation formelle autrement dit «le signifié d'un énoncé source étant repris dans un énoncé nouveau en des termes et des structures différentes » (Rabatel, à paraître), la reformulation établit une équivalence sémantique ou pragmatique entre deux énoncés contigus ou problématise la relation des 
choses et des mots pour les dire si on considère que la reformulation peut aussi établir directement la relation entre le référent et les mots qui le désignent.

En lien avec la paraphrase, la reformulation est définie par F. Neveu (2004 : 251) comme «la variante paraphrastique d'un segment linguistique, ou plus largement d'un énoncé, dans laquelle des modifications formelles sont opérées à des fins de spécification du sens ou de la référence ». La paraphrase qui est à la base de la définition de la reformulation a des affinités, qui ne sont pas seulement de paronymie accidentelle, avec la périphrase, en ce qu'elles servent toutes deux le processus de nomination ${ }^{1}$ du référent par une opération de substitution extensive, saisie à un point différent de sa réalisation.

La paraphrase propose la substitution, la périphrase l'impose. Je m'intéresserai dans un premier temps à la reformulation du nom commun exogène avant de me demander si le traitement du nom propre de lieux dans le récit de voyage pose des questions spécifiques. La reformulation de ce type de nom propre qu'on pourra assimiler à l'antonomase réalise un processus paraphrastique ou périphrastique mais surtout illustre un cas de paraphrase inclusive.

J'envisagerai ces trois procédés dans un continuum dynamique, spécifique du récit de voyage, à partir de la reformulation du nom commun exogène et du nom propre de lieu, en observant comment la reformulation peut devenir figurale. L'observatoire choisi est constitué d'exemples attestés, extraits du genre récit de voyage, tel qu'il est représenté dans la base Frantext catégorisée : le genre ainsi identifié compte 34 textes $^{2}$.

\section{La reformulation paraphrastique}

Les deux processus de la périphrase et de la paraphrase présentent des points de contact. Si la paraphrase est absente de la Pragmatique des figures du discours (Bonhomme, 2005), les deux paronymes trouvent place dans le Dictionnaire des procédés littéraires de B. Dupriez ainsi que dans l'inventaire des figures du discours établi par P. Fontanier. Les rhétoriciens s'accordent pour voir dans la périphrase une figure interprétable en termes de

\footnotetext{
1 Voir P. Siblot (2001). La nomination est perçue comme une approche dynamique de l'actualisation, par opposition à la dénomination qui relève du plan statique de la langue. « La dénomination relève d'une approche statique établie au plan de la langue, alors que la nomination désigne un acte de parole contextualisé, saisi dans la dynamique de l'actualisation ».

2 FRANTEXT - Base textuelle Frantext, ATILF (CNRS \& Université de Lorraine), http://www.frantext.fr. Toutes les références des citations sont données dans les éditions déposées dans Frantext.
} 
substitution par extension par rapport à la dénomination immédiate. La périphrase B d'un mot A est, si on veut retenir une définition linguistique, « un groupe non codé de plusieurs mots, qui a le même designatum ou le même signifié $»^{3}$ que le mot. P. Fontanier encore la définit comme figure d'emphase et la rapproche de la paraphrase de même que R. Martin (1983) voit dans la substitution périphrastique un type de paraphrase ${ }^{4}$.

Toutefois, la reformulation, choisie comme procédé englobant, se décline en différents types et tous n'utilisent pas la paraphrase : la reformulation paraphrastique exclut de fait la reformulation corrective réalisée par la forme prototypique «ou plutôt » qui règle la production de tout énoncé quel qu'il soit et traduit l'ajustement des mots aux choses :

[1] Quoi qu'il en soit, l'aspect de ces fenêtres est aussi joli qu'original. Le grenier qui constitue l'étage supérieur est une galerie, ou plutôt une suite de fenêtres rapprochées et copiées exactement sur celles qui forment le couronnement de la Lonja. (G. Sand, Un hiver à Majorque [1842], Paris, Glénat, 1993, p. 68) 5 .

La reformulation qui caractérise le récit de voyage est celle que j'appellerai alternative, c'est-à-dire celle qui propose une substitution, un autre énoncé possible qui n'efface pas la première occurrence. Elle établit l'équivalence sémantique entre deux énoncés successifs qui sont donnés comme substituables l'un à l'autre, envisagés pour ainsi dire comme synonymes, si on considère que la synonymie est validée par l'évaluation des commutations possibles dans plusieurs énoncés. Un terme peut remplacer l'autre dans un grand nombre de contextes, avec ici la particularité de l'équivalence interlinguale autorisée par le transfert assuré entre le nom commun exogène et sa conversion. Les deux énoncés appartiennent à deux langues différentes.

Par comparaison avec la reformulation corrective qui confère la primauté à la seconde occurrence présentée comme meilleure expression puisqu'elle arrive en seconde position dans la chaîne linéaire du texte, celle qui est dite paraphrastique se caractérise par deux critères distinctifs. Le premier est

\footnotetext{
${ }^{3}$ J. Rey-Debove (1979).

${ }^{4}$ L'énoncé définitionnel est par nature paraphrastique.

P. Bacry (2017 rééd.). Paraphrase : Expression développée permettant de désigner une réalité sans la nommer précisément mais en indiquant certaines de ses caractéristiques.

B. Dupriez (2003 rééd.). Paraphrase: développement explicatif d'un texte / sorte d'amplification oratoire par laquelle on développe et on accumule dans une même phrase plusieurs idées accessoires.

P. Fontanier (2009 rééd.). Périphrase : au lieu d'un seul mot, on en met plusieurs qui forment le même sens. P. Fontanier (Ibid.) distingue la pronomination qui remplace un nom par un syntagme nominal étendu et la périphrase qui remplace une phrase simple par une phrase plus complexe.

${ }^{5}$ Les italiques dans les citations sont de mon fait.
} 
qu'aucune hiérarchie n'est établie entre les unités successives ; le second que l'ordre des unités reliées n'est pas forcément imposé et son choix, quand il est possible, oriente sa lecture.

Quand un marqueur est observable entre les deux énoncés, il s'agit alors de formes qui affichent leur dimension métalinguistique parce qu'elles utilisent le verbe « dire » : «c'est-à-dire », « autrement dit», « ce qui veut dire » :

[2] Le peintre y avait établi son enderoun, c'est-à-dire l'appartement de sa femme. (J.-A. De Gobineau [1876], Nouvelles asiatiques, Paris, Garnier, 1965, p. 128).

[3] Caïmacam, c'est-à-dire le gouverneur turc d'Akabah. (P. Loti, Le Désert [1805], Paris, Laffont, 1991, p. 396).

[4] Il est souvent question chez eux de massâs, c'est-à-dire de mangeurs de morts (A. Gide, Retour du Tchad, [1928], Paris, Gallimard, 1960, p. 900).

Le signe de l'alternative «ou » associe les deux termes de manière minimale dans l'exemple suivant :

[5] Cette délicieuse vallée est arrosée d'une source où les Turcs viennent s'enivrer d'eau, de fraîcheur, de l'odeur des roses, et des chants du bulbul ou rossignol. (A. De Lamartine, Souvenirs, impressions, pensées et paysages pendant un voyage en Orient (1832-1833) ou Notes d'un voyageur (1835), Paris, 1861, p. 409).

Les parenthèses peuvent suffire enfin à la traduction interlinguale :

[6] La lonja (bourse) du commerce. (Th. Gautier, Voyage en Espagne [1843], Paris, Gallimard, 1981, p. 404).

Avec un marqueur métalinguistique, l'orientation de la structure va de l'inconnu au connu. En revanche, la conjonction ou, ou encore les signes doubles de ponctuation, maintiennent l'alternative possible et l'aller-retour d'un terme à l'autre envisageable. On peut alors suivre l'orientation qui sert la réduction de l'inconnu au connu, aussi bien que l'ordre symétrique. Deux énoncés sont placés en parallèle et cette co-présence assure le maintien de l'alternative entre deux mots, entre deux univers ou plutôt ouvre sur un univers ramifié, à plusieurs facettes. La lecture linéaire est freinée par autant de « brèches $»^{6}$ discursives, en faveur d'un parcours vertical ou tabulaire.

Quel que soit l'ordre choisi, le second terme prend une valeur commentative fonctionnant anaphoriquement par rapport au précédent, autrement appelé l'énoncé-source, qui peut être envisagé comme seul

\footnotetext{
${ }^{6}$ J. Authier-Revuz (1995) : 309.
} 
référentiel. Cet «espace clos qui s'ouvre et qui se ferme $»^{7}$ présente momentanément une autre scène qui exhibe la reformulation, dans un but didactique affiché. C'est un mouvement de vulgarisation ou une dynamique lexicographique qui mène de l'inconnu au connu et ramène l'excentricité dans l'univers partagé par locuteur et lecteur, tous deux réunis par une norme d'expression commune présupposée. Ce mouvement, centripète, ramène l'autre vers soi ${ }^{8}$.

Quand le terme final est le terme exogène en revanche, la tournure s'apparente davantage à une glose savante, de type dénominatif. Le mouvement est cette fois centrifuge et ouvert :

[7] Sur un seul dromadaire je vois une famille entière placée dans une sorte de manne (choukdieh) (M. Du Camp, Le Nil, Égypte et Nubie [1854], Paris, Hachette, 1877, p. 277).

[8] Celui des pages ou icoglans du grand sérail. (A. De Lamartine, Souvenirs, impressions, pensées et paysages pendant un voyage en Orient (1832-1833) ou Notes d'un voyageur [1835], Paris, Calmann-Lévy, 1861, 419).

La reformulation paraphrastique se spécialise dans sa fonction pédagogique ou didactique quand elle prend pour modèle le glossaire - le mot étranger est alors placé en première position - ou bien dans un rôle savant quand elle introduit le mot étranger placé en seconde position dans la sphère du connu, par un processus de conversion anticipé (exemples [7] et [8]).

Les limites de la reformulation dans le récit de voyage sont atteintes quand, en voulant traduire l'autre, l'énonciateur est contraint à un remodelage permanent qui confine à l'altération. Un point de vue sous-jacent anime les procédés de nomination?

À redire la même chose, on finit par dire autre chose - au terme d'un processus continu de déformations négligeables, voire imperceptibles ${ }^{10}$.

La reformulation opère un détournement même des outils dont la fonction a priori est d'explicitation pour servir l'expression d'un point de vue voire d'un jugement.

[9] Nous arrivâmes à une espèce de kan, c'est-à-dire à une masure à demi détruite, où un pauvre arabe cultive quelques figuiers et quelques courges, entre les fentes des rochers, auprès d'une fontaine. (A. De Lamartine, Souvenirs d'un Voyage en Orient [1835], Paris, Calmann-Lévy, p. 301).

\footnotetext{
${ }^{7}$ S. Boucheron-Pétillon (2002) : 97.

${ }^{8}$ Voir J. Authier-Revuz (1995) : 374.

${ }^{9}$ C. Fuchs (1982) : 63-4.

${ }^{10}$ C. Fuchs (1982) : 49-50.
} 
La reformulation est évaluation et non plus explicitation. En adoptant les outils canoniques de l'explicitation, la paraphrase est détournée de sa valeur explicative pour prendre un sens argumentatif voire esthétique dans l'extrait [10]:

[10] Les susceptibilités de la sérénade se sont beaucoup adoucies, et chacun peut rascar el jamon (gratter le jambon) sous la muraille de sa belle en toute tranquillité d'esprit.

(Th. Gautier, Voyage en Espagne [1843], Paris, Gallimard, 1981, p. 259).

La traduction littérale de l'expression lexicalisée dans la langue source ne joue aucun rôle informatif puisqu'elle exhibe un sens propre qui n'est pas celui activé dans l'expression originelle. La reformulation opacifie alors le sens du texte en y superposant une autre image, en faveur du pittoresque et en activant le potentiel esthétique de la forme.

\section{Le détour périphrastique ${ }^{11}$}

Les indices de l'approximation utilisés dans certains cas par la paraphrase explicative témoignent du caractère toujours imparfait de la reformulation. La paraphrase révèle le «caractère mouvant et subjectif des frontières que chacun établit entre le même et l'autre $»^{12}$. L'approximation s'accompagne le plus souvent d'un développement périphrastique qui élude le terme dénominatif exact, quelquefois parce qu'il n'est pas connu tout simplement.

La périphrase est, comme la paraphrase, une forme d'amplification mais elle est définie comme « détour désignatif ${ }^{13}$, comme «désignation indirecte et descriptive d'un référent en plusieurs mots $»^{14}$. On pourrait inscrire la paraphrase et la périphrase dans un mouvement graduel: la paraphrase maintient la coprésence des deux unités, dont une seule assure la référenciation et l'autre fonctionne comme apport explicatif; la périphrase remplace la dénomination exacte, par un procédé de contournement du terme dénominatif, et remplit le rôle de référenciation. Les exemples suivants [11] à [13] emploient tous une tournure d'approximation « une sorte de ». La périphrase s'appuie sur un modèle présent dans la mémoire endogène, un prototype au sens linguistique du terme: « un fourgon, un tabernacle, un bois ॥, trois concepts auxquels des propriétés stables sont présupposées s'associer. Le développement périphrastique à la droite de cette nouvelle

\footnotetext{
${ }^{11}$ Ce second point est volontairement moins développé. Il fera l'objet d'un autre article à venir.

${ }^{12}$ C. Fuchs $(1982): 50$.

${ }^{13}$ M. Bonhomme (2005).

${ }^{14}$ C. Fromilhague (1995) : 73.
} 
dénomination modalisée, sous la forme d'une relative adjective, ajuste ces modèles abstraits à la description de la réalité exogène.

[11] En nous promenant le soir sur le bord de la Vilaine, du côté des ponts, nous avons vu une sorte de long fourgon où l'on entrait par un escalier à double rampe et qui avait, le long de sa caisse, de petites fenêtres carrées à rideaux de coton rouge. (G. Flaubert, Par les champs et par les grèves [1848], Paris, Les belles Lettres, 1948, p. 405).

[12] J'ai devant moi un escalier de quelques marches, montant à une sorte de tabernacle qui me paraît chargé d'objets d'un art presque inconnu. (P. Loti, Les derniers jours de Pékin [1902], Paris, Laffont, p. 1088).

[13] Une heure de course à toutes jambes, et enfin voici devant moi cette Shiba étonnante. Au milieu de la ville, c'est une sorte de bois sacré qui garde du recueillement et du mystère sous les cèdres noirs aimés des dieux. (P. Loti, Japoneries d'automne [1889], Paris, Laffont, 1991, p. 148).

Pour l'exemple [13], on peut hésiter entre un développement paraphrastique qui renvoie au terme «shiba» présent dans le cotexte gauche et une périphrase désignative si on considère la distribution dans deux phrases distinctes du terme-source et de l'énoncé-cible suffisante pour garantir leur disjonction, tous deux assurant une fonction désignative autonome.

C'est une lecture à rebours quelquefois qui peut faire réinterpréter une périphrase comme paraphrase, dès que le terme exogène apparaît, inséré au cours de la séquence descriptive :

[14] Les femmes portent une sorte de guimpe blanche en dentelle ou en mousseline, appelée rebozillo, composée de deux pièces superposées. (G. Sand, Un hiver à Majorque [1842], Paris, Glénat, 1993, p. 119).

L'énoncé est interrompu par le terme exogène intercalé et le segment qui précède peut être interprété comme une paraphrase anticipée de ce terme.

Avec la reformulation du nom propre de lieux, on pourra retrouver cette double réalisation, sous la forme d'un énoncé paraphrastique ou périphrastique.

\section{La reformulation du nom propre de lieux - l'antonomase}

Le nom propre de lieux est au cœur du récit viatique, à tel point qu'on a pu proposer l'appellation de « roman géographique » ${ }^{15}$ sur le modèle de « roman historique » pour le qualifier.

\footnotetext{
${ }^{15}$ Voir V. Magri (2009). Le Voyage à pas comptés. Pour une poétique du récit de voyage au $X I X^{e}$ siècle, Paris, Champion.
} 
Précisons d'abord de quel emploi du nom propre il va être question. On sait que les principaux critères sémantiques et canoniques du nom propre retenus par contraste avec le nom commun sont la priorité de la fonction désignative qu'il assure, l'absence de connotation, le renvoi à la connaissance du référent.

On s'intéresse ici aux emplois non prototypiques du nom propre, à savoir ceux où la fonction désignative ${ }^{16}$ n'est pas prioritaire. Le nom propre extrait de l'univers connu est utilisé pour dénommer ou décrire un toponyme exogène. Il s'agit pour ainsi dire d'une reformulation à double détente : on transcrit la réalité étrangère - première (re)formulation - à l'aide de la reformulation d'un toponyme du pays d'origine ; celui-ci est détourné de sa fonction désignative première. Dans les exemples qui sont observés et qui correspondent à ce que certains linguistes appellent le nom propre modifié, ce dernier acquiert une "fonction non plus distinctive identifiante, mais descriptive, classifiante ou caractérisante $»^{17}$. N. Laurent (2015) établit une typologie quant à la modification du nom propre: sur le plan syntaxique lorsque le nom est accompagné d'une détermination quelle qu'elle soit, sur le plan sémantique si le contenu du nom propre est modifié, sur le plan référentiel enfin si le lien avec le référent initial est modifié.

$\mathrm{Au}$ niveau microstructural, il s'agit de repérer les constructions syntaxiques qui assurent ce détournement. Ces signaux syntaxiques regroupent l'emploi du déterminant à la gauche du nom propre, les expansions réalisées sous la forme d'un complément de nom ou d'un adjectif, les marqueurs d'approximation, autant de constructions qui suspendent la «fonction d'opérateur d'individualisation qui caractérise les noms propres ${ }^{18}$ et le font entrer dans un réseau sémantique dont il est à l'origine exclu, structuré par des relations en particulier de synonymie, voire d'antonymie et de polysémie :

[15] La Belgique est une Lombardie dont Anvers est la Venise. (J. Michelet, Sur les chemins de l'Europe [1874], Paris, Marpon et Flammarion, 1893, p. 229)

[16] [Le cratère de Rano-Kaou] C'est un Colisée immense et magnifique dans lequel manœuvrerait aisément toute une armée. (P. Loti, L'île de Pâques [1899], Paris, Laffont, 1991, p. 23)

L'exemple [15] offre une antonomase redoublée, exprimée une première fois sous la forme d'un complément attributif : le nom propre «Lombardie » est

\footnotetext{
${ }^{16} \mathrm{C}$ 'est la fonction désignative qui justifie l'appellation de «désignateur rigide » de Kripke, ie qui renvoie au même objet dans le monde réel et dans le monde construit, sans le décrire ou le classifier.

${ }^{17}$ K. Jonasson (1994) : 172.

${ }^{18}$ M.-N. Gary-Prieur (2005) : 53.
} 
précédé du déterminant indéfini qui fonctionne comme indicateur de son emploi détourné. La seconde occurrence se rattache syntaxiquement à ce nom propre, ce qui justifie l'emploi de l'article défini à la gauche de "Venise », lui aussi en emploi détourné.

Dans l'exemple [16], le Colisée n'est pas à proprement parler un toponyme mais, du fait de sa notoriété, il est entré dans la mémoire collective et connaît un emploi similaire. Le déterminant indéfini incongru pour le désigner opère comme une extraction d'un individu présenté comme exemplaire d'une classe. On peut hésiter sur la valeur sémantique des adjectifs qui complètent le nom propre: sont-ils non essentiels ou, au contraire, restrictifs et nécessaires à la complétude sémantique du terme support? J'opterais pour la valeur simplement caractérisante des adjectifs à supposer qu'il s'agit là de propriétés quasi définitoires du Colisée dans l'imaginaire collectif. Néanmoins, l'effet de redondance des adjectifs qui découle de cette interprétation est atténué par le fait que ces adjectifs pointent les propriétés communes et sélectionnées entre le référent comparé, "le cratère », et le comparant, le Colisée. La relative subséquente peut être envisagée comme non essentielle : elle sert d'expansion explicative aux épithètes précédentes, amplifiant l'image visuelle à créer dans l'imaginaire du lecteur.

Le syntagme est quelquefois complété par un complément restrictif à droite qui crée une nouvelle entité.

[17] En ce moment, j'ai eu l'idée d'une ville terrible, de quelque ville épouvantable et démesurée, comme serait une Babylone ou une Babel de cannibales où il y aurait des abattoirs d'hommes. (G. Flaubert, Par les champs et par les grèves [1848], Paris, Les Belles Lettres, 1948, p. 289)

L'expansion à droite bride la simple interprétation désignative ou dénominative du nom propre ${ }^{19}$ et fonctionne comme instruction à construire l'interprétation métaphorique. Le complément en de, «de cannibales », complété par la relative, joue le rôle d'une enclosure en ce sens qu'il « neutralise les incompatibilités entre les domaines sémantiques » (Rastier, 1987, 62) et crée une nouvelle entité.

Un marqueur d'approximation s'associe encore à l'expansion adjective restrictive à droite dans l'exemple suivant, pour moduler l'incongruité du toponyme modifié :

[18] Parfois une plante grimpante à larges et belles feuilles retombait et formait cadre ou feston à ces étranges parois en ruine, faisant valoir la richesse et la sonorité de leurs tons. On eût dit une sorte de Pompéi nègre. (A. Gide, Voyage au Congo [1927], Paris, Gallimard, 1960, p. 785).

\footnotetext{
${ }^{19}$ Voir K. Jonasson.
} 
Alors que le nom propre est considéré comme inapte à la fonction prédicative qui consiste à attribuer une propriété à un référent désigné, les emplois non prototypiques entrent dans des constructions syntaxiques qui confèrent une telle fonction au nom propre : attribut ${ }^{20}$ ou structure appositive. Les deux noms propres renvoient au même référent, l'un pouvant être interprété littéralement, l'autre métaphoriquement. Ces schémas syntaxiques correspondent à ce que $\mathrm{S}$. Leroy, après $\mathrm{K}$. Jonasson ${ }^{21}$, nomme l'antonomase in praesentia qui fonctionne, si je veux conserver la terminologie que j'ai choisie, comme une paraphrase définitionnelle ; ainsi en est-il des exemples d'antonomase en apposition dans les exemples suivants :

[19] Le pauvre évêque grec de Zarklé est d'une famille d'Alep, où il a passé sa vie dans l'élégance et la mollesse des mœurs de cette ville, l'Athènes de l'Asie. (A. De Lamartine, Souvenirs, impressions, pensées et paysages pendant un voyage en Orient (1832-1833) ou Notes d'un voyageur [1835], Paris, 1861, p. 248).

[20] Abyla, maintenant le Mont des Singes, est de l'autre côté en Afrique, tout près de Ceuta, possession espagnole, le Brest et le Toulon de la Péninsule, où l'on envoie les plus endurcis des galériens. (Th. Gautier, Voyage en Espagne [1843], Paris, Gallimard, 1981, p. 436).

[21] C'est la plaine d'Argos, cette Beauce de la Grèce, où les jeunes filles cueillent les feuilles de mûrier et sèment la graine du coton. (É. About, La Grèce contemporaine [1854], Paris, Hachette, 1858, p. 28).

La présence du complément de nom « de l'Asie », « de la Péninsule », « de la Grèce » permet d'ajuster le toponyme comparant au lieu exogène décrit, en ajoutant la précision sur le déplacement géographique à opérer et la relocalisation à réaliser.

De manière symétrique, l'antonomase in absentia correspond au fonctionnement par substitution de la périphrase, dans des structures à présentatif ou en fonction de sujet :

[22] [Le Liban] C'est une montagne solennelle comme son nom. Ce sont les Alpes sous le ciel de l'Asie, plongeant leurs cimes aériennes dans la profonde sérénité d'une éternelle splendeur. (A. De Lamartine, Souvenirs, impressions, pensées et paysages pendant un voyage en Orient (1832-1833) ou Notes d'un voyageur [1835], Paris, 1861, p. 177).

[23] Et cette Arcadie de la Judée réunit ainsi toujours, à la majesté et à la gravité des contrées montagneuses, l'image de la fertilité et de l'abondance variées de la terre. (A. De Lamartine, Ibid., p. 335)

\footnotetext{
${ }^{20}$ Voir K. Jonasson (1993).

${ }^{21}$ Voir Leroy (2004) :108-109, 123 et Jonasson (1993a et 1990).
} 
[24] Retournez-vous, le colossal manoir se dresse à pic sur la rivière, comme le Capitole sur le forum romanum. Mais ici, c'est le Colisée qui occupe la colline du Capitole. Vue moins sublime, mais douce et consolante de jeune vie, près de cette sombre antiquité. (J. Michelet, Sur les chemins de l'Europe. En Angleterre [1874], Paris, Marpon et Flammarion, 1893, p. 66)

Ce type d'antonomase illustre le processus de la catégorisation qui rapproche le fonctionnement du nom propre de celui du nom commun, pourvu d'un sens conceptuel. Le nom propre crée une « classe discursive », contingente et instable puisqu'elle est dépendante du contexte d'énonciation ${ }^{22}$. Cette classe est fondée sur des liens de similarité établis entre le référent initial et le référent discursif.

Ce sont les relations avec le référent initial qui sont problématisées et réévaluées sur le plan métonymique ou métaphorique. Sans revenir sur le débat qui a opposé les linguistes sur le sens du nom propre ${ }^{23}$, on retiendra que si le nom propre n'a pas de sens conceptuel codifié qui en ferait une unité de la langue, il a un sens de «nature instructionnelle » ${ }^{24}$ qui permet d'établir une relation de référence. Et, en raison de ce lien mémoriel avec le référent originel qui persiste quels que soient les emplois, on peut affirmer à la suite de plusieurs linguistes que le nom propre est associé à un contenu - (GaryPrieur 2005, 60) ou à un dossier pour reprendre le terme de P. Grice ; celuici correspond à un ensemble de connaissances encyclopédiques à acquérir ; autrement dit, le nom propre est associé à un ensemble de propriétés acquises par un lien mémoriel établi avec le référent originel, dès lors que l'acte de baptême a eu lieu.

L'essentiel du sens d'un nom propre est contenu dans un extralinguistique réel ou imaginaire, avec lequel il est pratiquement en relation de désignation directe; l'existence de ce sens suppose une connaissance directe du référent ou indirecte par le biais d'une description de type encyclopédique. [...] Le nom propre possède en réalité un potentiel de signification aussi riche et un rapport au sens au moins aussi complexe que le simple nom commun. (Ballard, 2001, p. 107)

Que la reformulation du nom propre relève d'un processus de paraphrase, quand est maintenue la coprésence des deux noms propres, ou d'un processus de périphrase, lorsque le nom propre qui intervient à titre de comparant remplace le comparé, l'antonomase inclut une autre paraphrase implicite, à lire dans le nom propre convoqué en emploi non prototypique. Une sélection

\footnotetext{
${ }^{22}$ Gary-Prieur (1994) : 79.

${ }^{23}$ Pour Kripke par exemple, le nom propre est vide de sens : une fois l'acte de baptême effectué, le nom propre repère un objet du monde. Pour Russel, Frege, Searle ou Strawson, le nom propre est une « description déguisée ».

${ }^{24}$ Gary-Prieur (2005) : 60. Ce sens de nature instructionnelle permet d'établir une relation de référence dans le cadre d'un énoncé.
} 
parmi les propriétés attribuées au référent initial est opérée en contexte. La part de ces connaissances qui est activée est instable et dépend du contexte où s'insère la reformulation du toponyme. Le toponyme reformulé assure une nomination renouvelée dans le cadre du récit.

Il faudrait, pour chaque antonomase, déchiffrer les relations qu'a établies l'énonciateur entre les deux toponymes, en fait retrouver le lien entre les deux référents, les deux objets du monde, instauré en discours. C'est sur le pari d'une même reconnaissance effectuée par le lecteur que reposent la validité et la lecture de ces expressions. Les propriétés du référent initial sont choisies en fonction d'un point de vue. Par exemple, le Colisée intervient à titre de comparant aussi bien dans l'exemple de Loti [16] que dans celui de Michelet [24] - l'immensité est la propriété commune retenue, exprimée dans le premier exemple, allusive dans le second. Devant un lieu étranger, un élément de la mémoire collective est activé qui permet d'établir une communauté culturelle avec le lecteur, en remodelant un toponyme endogène, grâce à la modification syntaxique de son expression linguistique et grâce à la mise en sourdine, au second plan, du lien avec le référent d'origine.

Le toponyme étranger peut encore être traduit et non plus seulement confronté à un toponyme endogène. Les frontières entre nom propre et nom commun s'effacent alors au profit d'une recréation lexicale. L'énonciateur active un potentiel sémantique indécelable, lié quelquefois à l'acte de baptême qui a prévalu à la création du nom propre comme dans l'exemple suivant :

[25] Le Puerto de los Perros (passage des chiens) est ainsi nommé parce que c'est par là que les Maures vaincus sortirent de l'Andalousie, emportant avec eux le bonheur et la civilisation de l'Espagne. (Th. Gautier, Voyage en Espagne [1843], Paris, Gallimard, 1981, p. 243).

Ce potentiel sémantique restauré se pare quelquefois d'une plus-value esthétique voire poétique, à la faveur de métaphores en sommeil et indétectables pour le lecteur: «Bir-El-Hamamat» est transposé en le puits des pigeons (M. Du Camp, Le Nil, op. cit., p. 271).

\section{Conclusion}

La reformulation, posée comme acte définitoire du récit de voyage, se module selon trois processus qui ont été observés au long d'un continuum dynamique comme manifestations du réglage du sens permanent.

La paraphrase propose une reformulation alternative qui maintient sur le même plan terme exogène et terme converti ; la périphrase remplace le terme exogène par un énoncé qui assure la désignation directe. L'antonomase est 
plus complexe : elle désigne l'objet du monde exogène, en l'occurrence le nom de lieu, par l'emploi d'un nom propre lui-même reformulé. Elle réalise une triple modification : syntaxique par les constructions spécifiques où elle fait entrer le nom propre, sémantique par la sélection qu'elle opère d'une part de son contenu, référentielle enfin puisqu'elle le détache de son référent d'origine. Dès lors, cette figure inclut encore un processus paraphrastique qui commente de manière explicite ou implicite le nom propre modifié, en pariant sur les capacités de décodage et d'interprétation du lecteur. La reformulation affirme, au travers de ces trois processus, sa dimension dialogique et sa place au cœur de ce triangle pragmatique dont il a été question en début d'étude. L'énonciateur veut imposer sa vision du monde dans une relation de coénonciation tandis que c'est la relation du langage au réel qui est repensée : le récit viatique est ce lieu où s'entremêlent deux mondes, où les notions mêmes ne peuvent se définir que dans un réseau associatif, construit sur des liens de contraste ou d'analogie, où le sens des mots est instable et relatif, comme la perception de son propre univers, confronté à l'altérité.

\section{Bibliographie}

Authier-Revuz J. (1995). Ces Mots qui ne vont pas de soi. Boucles réflexives et non-coïncidences $d u$ dire, Paris, Larousse.

Bacry P. (2017 rééd.). Les Figures de style. Et autres procédés stylistiques. Paris, Belin.

Ballard M. (1998). «La traduction du nom propre comme négociation », Palimpsestes, 11, 199223.

Bonhomme M. (2005). Pragmatique des figures de discours, Paris, Champion.

Boucheron-Pétillon (2002). Les Détours de la langue. Étude sur la parenthèse et le tiret double, Louvain, Paris, Peeters.

Dupriez B. (2003). Gradus. Les procédés littéraires, Paris, 10/18.

Fontanier P. (2009 rééd.). Les Figures du discours, Paris, Flammarion.

Fromilhague C. (1995). Les Figures de style, Paris, Nathan.

Fuchs C. (1982). La Paraphrase, Paris, PUF.

Gary-Prieur M.-N. (1994). Grammaire du nom propre, Paris, CNRS Editions.

Gary-Prieur M.-N. (2005). «Où il est montré que le nom propre n'est (presque) jamais modifié », Langue française, 146-2, 53-66.

Jonasson K. (1994). Le nom propre. Constructions et interprétations, Louvain-la-Neuve, Duculot.

Jonasson K. (1993). «Le nom propre en fonction d'attribut », Studier I Modern Sprãkvetenskap, 10, 132-153.

Jonasson K. (1993)a. «Le point d'ancrage référentiel des noms propres métaphoriques », in Actes du XIXe Congrès international de linguistique et de philologie romanes, Coruña, vol. 4, 330-342.

Jonasson K. (1991). « Les noms propres métaphoriques : construction et interprétation », Langue française $\mathbf{9 2}, 64-81$.

Jonasson K. (1990) « Métaphores in absentia et la lexicalisation des noms propres », in Actes du $\mathrm{XI}^{\mathrm{e}}$ Congrès des romanistes scandinaves, Trondheim 13/17, 261-271. 
Kleiber G. (1984), « Dénomination et relations dénominatives », Langages 76, 77-94.

Laurent N. (2013). «Le nom propre dans les Lettres de l'année 1671 de Mme de Sévigné » CÉRÉdI, 1, http://ceredi.labos.univ-rouen.fr/public/?le-nom-propre-dans-les-lettres-de.html.

Laurent N. \& Reggiani Ch. (2015). Seuils du nom propre, Paris, Lambert-Lucas.

Leroy S. (2004). De l'identification à la catégorisation. L'antonomase du nom propre en français, Louvain, Peeters. https://tel.archives-ouvertes.fr/tel-00086307/

Leroy S. (2002). « De Harpagon à un Harpagon : la question de la lexicalisation du nom propre en antonomase", Bulag 27 Les mots, leur sens, leur forme, leur création et leur reconnaissance, 119-133.

Magri V. \& Rabatel A. (2015). Répétitions et genres, Le Discours et la langue, 7.2.

Martin R. (1983). Pour une logique du sens, Paris, PUF.

Neveu F. (2004). Dictionnaire des sciences du langage, Paris, Armand Colin.

Noille C. «Rhétorique de la périphrase » in Le Mot propre et la périphrase. Du tour précieux à "l'objet tu », Classiques Garnier, 2014, 27-43.

Petit G. Langue française (2012/2), 174, La Dénomination.

Petit G., Haillet P., X-L. Salvador (2017). La Dénomination: Lexique et discours. Paris, Champion.

Rey-Debove J. (1979). Lexique sémiotique, Paris, PUF.

Siblot P. (2001). «De la dénomination à la nomination. Les dynamiques de la signifiance nominale et le propre du nom ", Cahiers de praxématique, Linguistique de la dénomination, 36. http://journals.openedition.org/praxematique/368, consulté le 29-12-2017. 